\title{
Performance Analysis of Multiple-Symbol Differential Detection for OFDM over Both Time- and Frequency-Selective Rayleigh Fading Channels
}

\author{
Akira Ishii \\ Department of Communications and Systems, University of Electro-Communications, 1-5-1 Chofugaoka, \\ Chofu-shi, Tokyo 182-8585, Japan \\ Email:a.ishii@ieee.org \\ Hideki Ochiai \\ Division of Physics, Electrical and Computer Engineering, Yokohama National University, 79-1 Tokiwadai, \\ Hodogaya-ku, Yokohama 240-8501, Japan \\ Email: hideki@ynu.ac.jp \\ Tadashi Fujino \\ Department of Communications and Systems, University of Electro-Communications, 1-5-1 Chofugaoka, \\ Chofu-shi, Tokyo 182-8585, Japan \\ Email:fujino@ice.uec.ac.jp
}

Received 28 February 2003; Revised 8 October 2003

\begin{abstract}
The performance of orthogonal frequency-division multiplexing (OFDM) system with multiple-symbol differential detection (MSDD) is analyzed over both time- and frequency-selective Rayleigh fading channels. The optimal decision metrics of timedomain MSDD (TD-MSDD) and frequency-domain MSDD (FD-MSDD) are derived by calculating the exact covariance matrix under the assumption that the guard time is longer than the delay spread, thus causing no effective intersymbol interference (ISI). Since the complexity of calculating the exact covariance matrix turns out to be substantial for FD-MSDD, we also develop a suboptimal metric based on the simplified covariance matrix. The comparative analysis between TD-MSDD and FD-MSDD suggests that the most significant improvement is achieved by the FD-MSDD with the optimal metric and a large symbol observation interval, since the time selectiveness of the channel has a dominant effect on the bit error rate of the OFDM system.
\end{abstract}

Keywords and phrases: orthogonal frequency-division multiplexing, multiple-symbol differential detection, time- and frequencyselective channels, Rayleigh fading.

\section{INTRODUCTION}

In mobile communications systems, there has been a growing demand for high data rate services such as video phone, high-quality digital distribution of music, and digital television terrestrial broadcasting (DTTB) [1]. In such systems, the delay spread of the channel becomes a major impairment to cope with, since it may cause a severe intersymbol interference (ISI). It is well known that the orthogonal frequency-division multiplexing (OFDM), which transmits the information symbols in parallel over a number of spectrally overlapping but temporally orthogonal subchannels [2], is an effective technique to combat the ISI. With a guard interval longer than the maximum delay spread of the channel, OFDM can effectively avoid the ISI with high spectral efficiency and reasonable complexity. However, the time-selective nature of the channel due to the Doppler shift also results in the loss of orthogonality among subcarriers, causing a considerable interchannel interference (ICI) [3].

When the time selectiveness of the channel becomes severe, that is, both amplitude and phase of the received signal vary fast, the reliable estimation of the channel state information (CSI) becomes challenging. In such cases, the differential detection (DD) in combination with OFDM may lead to a simple receiver structure, eliminating the need for complex channel estimation. In general, however, the DD suffers from a performance penalty, compared to coherent detection with perfect CSI over an additive white Gaussian noise 
(AWGN) channel. In order to reduce this gap between the coherent detection and conventional DD, the multiple-symbol differential detection (MSDD) has been introduced for $M$ ary phase-shift keying (MPSK) signals over the AWGN channel in [4]. Making a joint decision on a block of $N_{M}$ consecutive information symbols based on $N_{M}+1$ received samples as opposed to conventional symbol-by-symbol detection, MSDD can asymptotically achieve the performance of the coherent detector. Since the conventional DD or MSDD relies on the time-invariant nature of the channel impulse response over adjacent symbols, its performance will be considerably degraded when the channel is time selective, which results in an irreducible error floor. To cope with this time variance, MSDD has been modified in $[5,6]$. Its decision metric utilizes the covariance matrix conditioned on the transmitted information symbol sequence.

For OFDM, the DD can be applied over time domain, frequency domain, or both. Because of the long symbol duration, the performance of the time-domain DD (TD-DD) may be mostly affected by the time-selective fading. On the other hand, the performance of the frequency-domain DD (FD-DD) may also depend on the frequency-selectiveness of the channel associated with delay spread $[7,8]$. In $[8,9,10]$, the bit error rate (BER) performance of TD-DD and FD-DD has been theoretically analyzed over time- and frequencyselective Rayleigh fading channels, including the effects of the ISI caused by the delay spread longer than the guard time. In [11], the performance of MSDD with coded modulation has been studied in terms of channel capacity over quasistatic Rayleigh fading channels with OFDM scenario and ideal interleaving.

In this paper, the performance of MSDD combined with OFDM is analyzed over time- and frequency-selective Rayleigh fading channels. Assuming the guard time is longer than the delay spread, we derive the optimal decision metrics. Furthermore, we study the theoretical BER performance of the MSDD for OFDM by extending the result of [6]. Our approach is based on the truncated union bound, which counts only dominant terms of the pairwise error probability (PEP) in the union bound. Based on these analytical results, we compare TD-MSDD and FD-MSDD in terms of irreducible BER behavior for high signal-to-noise ratio (SNR).

The paper is organized as follows. After the description of the system model considered throughout the paper in Section 2, we describe the proposed metrics of TD-MSDD and FD-MSDD in Section 3. The bit error probability based on these metrics is studied in Section 4. Section 5 is devoted to a comparative study on the theoretical and simulation results of the MSDD with the various decision metrics developed in the paper. Finally, concluding remarks are given in Section 6.

\section{SYSTEM MODEL}

\subsection{OFDM with differential encoding}

The discrete-time baseband equivalent model of the system under consideration is described in Figure 1. Informa-

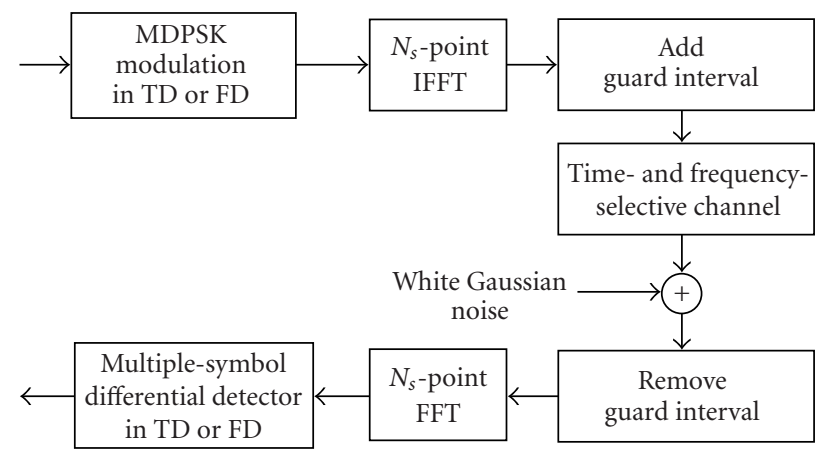

FIGURE 1: The discrete-time baseband equivalent model of OFDM with MSDD.

tion bits are Gray mapped onto MPSK and let $c_{i}(n)=$ $\exp \left(j \theta_{i}(n)\right)$, where $\theta_{i}(n) \in\{(2 \pi m) / M, m=0,1, \ldots, M-$ $1\}$, denote the information symbol prior to the differential encoding, which will be assigned on the $n$th subcarrier of the $i$ th OFDM symbol with $N_{s}$ subcarriers. Information symbols are assumed to be independent and identically distributed (i.i.d.). For TD-(MS)DD, information symbols are differentially encoded over the consecutive OFDM symbols with the same subcarrier index $n$. For FD-(MS)DD, on the other hand, information symbols are differentially encoded over the adjacent subcarriers within the same OFDM symbol index $i$. The differentially encoded symbol $s_{i}(n)$ in each domain can be thus expressed as

$$
s_{i}(n)= \begin{cases}c_{i}(n) s_{i-1}(n), & \text { in } \mathrm{TD} \\ c_{i}(n) s_{i}(n-1), & \text { in } \mathrm{FD}\end{cases}
$$

where $s_{i}(n) \in\{\exp (j 2 \pi m / M), m=0,1, \ldots, M-1\}$. The symbol transmitted on the $n$th subcarrier of the $i$ th OFDM symbol is given by

$$
a_{i}(n)=\sqrt{E_{s}} s_{i}(n), \quad n=0,1, \ldots, N_{s}-1,
$$

where $E_{s}$ denotes the signal energy per subcarrier symbol.

The complex sequence $a_{i}(n), n=0,1, \ldots, N_{s}-1$, is modulated by the $N_{s}$-point inverse discrete Fourier transform (IDFT) to yield $N_{s}$ time-domain samples corresponding to the $i$ th OFDM symbol. Let $T_{s}$ denote the Nyquist interval between the output samples. Thus, the OFDM symbol length without guard interval is given by $N_{s} T_{s}$. After the insertion of the guard interval, the transmitted baseband sequence of the $i$ th OFDM symbol can be expressed as

$$
x_{i}^{g}(k)=\frac{1}{\sqrt{N_{s}}} \sum_{n=0}^{N_{s}-1} a_{i}(n) e^{j\left(2 \pi n k / N_{s}\right)} \quad \text { for }-G \leq k \leq N_{s}-1,
$$

where the initial $G$ samples of $x_{i}^{g}(k), k=-G,-G+1, \ldots,-1$, constitute the guard interval. Assuming that $x_{i}^{g}(k)$ is zero for $k<-G$ and $k \geq N_{s}$, the total transmitted baseband sequence 
is written as

$$
x(k)=\sum_{i=-\infty}^{\infty} x_{i}^{g}\left(k-i\left(N_{s}+G\right)\right) .
$$

\subsection{Channel model and received baseband sequence}

We assume that the channel is subject to a wide-sense stationary uncorrelated scattering (WSSUS) Rayleigh fading [12] and is modeled as a time-variant tapped delay line with fixed tap spacing $T_{s}$, each tap having Jakes power spectrum [13]. Provided that the maximum delay of the channel impulse response $T_{m}$ does not exceed $M_{p} T_{s}$ for some integer $M_{p}$, the received baseband sequence assuming perfect synchronization can be expressed as

$$
r(k)=\sum_{i=-\infty}^{\infty} \sum_{m=0}^{M_{p}-1} h_{m}(k) x_{i}^{g}\left(k-m-i\left(N_{s}+G\right)\right)+n(k),
$$

where $n(k)$ is the sample of an AWGN process. Then, the $i$ th received OFDM symbol can be given by $r_{i}(k)=r\left(i\left(N_{s}+G\right)+\right.$ $k)$ for $-G \leq k \leq N_{s}-1$. Assuming that $T_{m}$ does not exceed $G T_{s}$, the $r_{i}(k)$ after eliminating the initial $G$ guard samples can be expressed as

$$
r_{i}(k)=\sum_{m=0}^{M_{p}-1} h_{m, i}(k) x_{i}^{g}(k-m)+n_{i}(k) \quad \text { for } 0 \leq k \leq N_{s}-1,
$$

where $h_{m, i}(k)=h_{m}\left(i\left(N_{s}+G\right)+k\right)$. The demodulator performs DFT on $\left\{r_{i}(k), 0 \leq k \leq N_{s}-1\right\}$, producing the output [14]

$$
\begin{aligned}
R_{i}(l)= & \frac{1}{N_{s}}\left(\sum_{k=0}^{N_{s}-1} \sum_{m=0}^{M_{p}-1} h_{m, i}(k) e^{-j\left(2 \pi l m / N_{s}\right)}\right) a_{i}(l) \\
& +\frac{1}{N_{s}} \sum_{n \neq l} a_{i}(n) \sum_{k=0}^{N_{s}-1} \sum_{m=0}^{M_{p}-1} h_{m, i}(k) e^{-j\left(2 \pi n m / N_{s}\right)} e^{j\left(2 \pi k(n-l) / N_{s}\right)} \\
& +\frac{1}{\sqrt{N_{s}}} \sum_{k=0}^{N_{s}-1} n_{i}(k) e^{-j\left(2 \pi k l / N_{s}\right)} \\
= & H_{i}(l) a_{i}(l)+C_{i}(l)+W_{i}(l), \quad \text { for } 0 \leq l \leq N_{s}-1 .
\end{aligned}
$$

Here, $R_{i}(l)$ denotes the received symbol on the $l$ th subcarrier of the $i$ th OFDM symbol. In $(7), H_{i}(l), C_{i}(l)$, and $W_{i}(l)$ are the multiplicative distortion, the ICI, and the AWGN, respectively, on the $l$ th subcarrier of the $i$ th OFDM symbol. Based on $R_{i}(l)$, a multiple-symbol differential detector in each domain makes a decision on the estimated information symbols, which is described in the next section.

\section{OPTIMAL AND SUBOPTIMAL METRICS}

\subsection{Multiple-symbol differential detection}

Following the basis on the MSDD system in $[4,5,6]$, we rewrite the transmitted complex sequence in (2) as

$$
\begin{aligned}
& a_{i+d}(l)=\sqrt{E_{s}} s_{i}(l) z_{d, i}(l), \quad \text { in TD, } \\
& a_{i}(l+d)=\sqrt{E_{s}} s_{i}(l) z_{d, i}(l), \quad \text { in } \mathrm{FD},
\end{aligned}
$$

where

$$
\begin{aligned}
& z_{d, i}(l)=\left\{\begin{array}{ll}
1, & \text { for } d=0, \\
\prod_{j=1}^{d} c_{i+j}(l), & \text { for } 1 \leq d \leq N_{M},
\end{array}\right. \text { in TD, } \\
& z_{d, i}(l)=\left\{\begin{array}{ll}
1, & \text { for } d=0, \\
\prod_{j=1}^{d} c_{i}(l+j), & \text { for } 1 \leq d \leq N_{M},
\end{array} \quad\right. \text { in FD, }
\end{aligned}
$$

and $N_{M}$ denotes the observation interval of the information symbols. Note that with this definition of $N_{M}$, the conventional DD corresponds to the case with $N_{M}=1$. Also, apparently, we have $z_{d, i}(l) \in\{\exp (j 2 \pi m / M), m=0,1, \ldots, M-1\}$.

The received symbols in (7) are divided into a detection block that consists of $\left(N_{M}+1\right)$ symbols as

$$
\mathbf{R}_{i}(l)= \begin{cases}\left(R_{i}(l), R_{i+1}(l), \ldots, R_{i+N_{M}}(l)\right)^{t}, & \text { in TD, } \\ \left(R_{i}(l), R_{i}(l+1), \ldots, R_{i}\left(l+N_{M}\right)\right)^{t}, & \text { in FD, }\end{cases}
$$

where, throughout the paper, the notations $(\cdot)^{t}$ and $(\cdot)^{\dagger}$ are used to denote the transpose and the Hermitian transpose, respectively. The column vector $\mathbf{R}_{i}(l)$ is input to a multiple-symbol differential detector implemented based on maximum-likelihood sequence estimation (MLSE). The MLSE detects the most likely estimated information symbol sequence

$$
\hat{\mathbf{c}}_{i}(l)= \begin{cases}\left(\hat{c}_{i+1}(l), \ldots, \hat{c}_{i+N_{M}}(l)\right), & \text { in TD, } \\ \left(\hat{c}_{i}(l+1), \ldots, \hat{c}_{i}\left(l+N_{M}\right)\right), & \text { in FD, }\end{cases}
$$

from all $M^{N_{M}}$ possible $N_{M}$-length sequences. As shown in [6], this is accomplished by selecting the sequence $\hat{\mathbf{c}}_{i}(l)$ of which the metric

$$
M\left(\hat{\mathbf{c}}_{i}(l)\right)=\mathbf{R}_{i}(l)^{\dagger} \hat{\boldsymbol{\Phi}}_{\mathbf{R}_{i}(l)}^{-1} \mathbf{R}_{i}(l)
$$

is the smallest, where $\hat{\boldsymbol{\Phi}}_{\mathbf{R}_{i}(l)}$ is a covariance matrix of $\mathbf{R}_{i}(l)$ conditioned on $\hat{\mathbf{c}}_{i}(l)$. It should be noted that the complexity of MSDD increases exponentially with $M^{N_{M}}$. In the following, we derive the covariance matrix for each case.

\subsection{Covariance matrix in time-domain MSDD}

The covariance of $R_{i}(l)$ in (7) can be written as

$$
\begin{aligned}
E\left[R_{i}(l) R_{i+\alpha}^{*}(l)\right]=E[ & a_{i}(l) H_{i}(l) H_{i+\alpha}^{*}(l) a_{i+\alpha}^{*}(l) \\
& +a_{i}(l) H_{i}(l) C_{i+\alpha}^{*}(l)+a_{i+\alpha}^{*}(l) H_{i+\alpha}^{*}(l) C_{i}(l) \\
& \left.+C_{i}(l) C_{i+\alpha}^{*}(l)+W_{i}(l) W_{i+\alpha}^{*}(l)\right],
\end{aligned}
$$

where the notation $E[\cdot]$ and * * are used to denote the expectation and complex conjugate, respectively. For uncorrelated and isotropic scattering, the correlation of the tap coefficients 
is expressed, by definition, as

$$
\begin{aligned}
& E\left[h_{m, i}(k) h_{m^{\prime}, i+\alpha}^{*}\left(k^{\prime}\right)\right] \\
& \quad=\sigma_{m}^{2} J_{0}\left(2 \pi f_{D} T_{s}\left(k-k^{\prime}-\alpha\left(N_{s}+G\right)\right)\right) \delta_{m, m^{\prime}},
\end{aligned}
$$

where $\sigma_{m}^{2}$ is the average power of the $m$ th channel tap, $J_{0}(\cdot)$ is the zeroth-order Bessel function of the first kind, $f_{D}$ is the maximum Doppler frequency, and $\delta_{m, m^{\prime}}$ is the Kronecker delta function. By normalizing the average power of each path such that $\sum_{m=0}^{M_{p}-1} \sigma_{m}^{2}=1$, the correlation of the multiplicative distortion is expressed as

$$
\begin{aligned}
\phi_{t}(\alpha) & \equiv E\left[H_{i}(l) H_{i+\alpha}^{*}(l)\right] \\
& =\frac{1}{N_{s}^{2}} \sum_{k=0}^{N_{s}-1} \sum_{k^{\prime}=0}^{N_{s}-1} J_{0}\left(2 \pi f_{D} T_{s}\left(k-k^{\prime}-\alpha\left(N_{s}+G\right)\right)\right) .
\end{aligned}
$$

Due to the assumption of the statistical independence of the information symbols, we have $E\left[a_{i}(l) a_{i^{\prime}}^{*}\left(l^{\prime}\right)\right]=E_{s} \delta_{i, i^{\prime}} \delta_{l, l^{\prime}}$, which yields

$$
E\left[a_{i}(l) H_{i}(l) C_{i+\alpha}^{*}(l)\right]=E\left[a_{i+\alpha}^{*}(l) H_{i+\alpha}^{*}(l) C_{i}(l)\right]=0 .
$$

As shown in [3], for sufficiently large $N_{s}$, the central limit theorem can be invoked and the ICI can be modeled as a complex Gaussian random process with zero mean. Then the correlation of the ICI can be obtained as

$$
\begin{aligned}
& E\left[C_{i}(l) C_{i+\alpha}^{*}(l)\right] \\
& \quad=\left(E_{s}-\frac{E_{s}}{N_{s}^{2}}\left(N_{s}+2 \sum_{k=1}^{N_{s}-1}\left(N_{s}-k\right) J_{0}\left(2 \pi f_{D} T_{s} k\right)\right)\right) \delta_{0, \alpha} \\
& \quad \equiv \sigma_{\mathrm{ICI}}^{2} \delta_{0, \alpha},
\end{aligned}
$$

where $\sigma_{\mathrm{ICI}}^{2}$ is the variance of the ICI.

The correlation of the AWGN is given by

$$
E\left[N_{i}(l) N_{i+\alpha}^{*}(l)\right]=N_{0} \delta_{0, \alpha}
$$

where $N_{0}$ is the one-sided power spectral density of the AWGN process.

Recognizing that the covariance matrix of arbitrary $R_{i}(l)$ denoted by $\boldsymbol{\Phi}_{\mathbf{R}_{i}(l)}$ is irrelevant to the index $l$, and using (13), (14), (15), (16), (17), and (18), one can easily show that

$$
\boldsymbol{\Phi}_{\mathbf{R}_{i}}=\mathbf{A}_{i} \boldsymbol{\Phi}_{t} \mathbf{A}_{i}^{\dagger}+\left(N_{0}+\sigma_{\mathrm{ICI}}^{2}\right) \mathbf{I},
$$

where $\mathbf{A}_{i}=\operatorname{diag}\left(a_{i}, a_{i+1}, \ldots, a_{i+N_{M}}\right)$ is a diagonal matrix, $\boldsymbol{\Phi}_{t}$ is the covariance matrix of the multiplicative distortion of which the $(\beta, \gamma)$ th element can be expressed as $\phi_{t}(\gamma-\beta)$ defined in (15), and I is the identity matrix of size $N_{M}+1$. With
$\mathbf{A}_{i} \mathbf{A}_{i}^{\dagger}=E_{s} \mathbf{I}$ and (8), (19) can be rewritten as

$$
\boldsymbol{\Phi}_{\mathbf{R}_{i}}=\mathbf{Z}_{i}\left(E_{s} \boldsymbol{\Phi}_{t}+\left(N_{0}+\sigma_{\mathrm{ICI}}^{2}\right) \mathbf{I}\right) \mathbf{Z}_{i}^{\dagger}
$$

where $\mathbf{Z}_{i}=\operatorname{diag}\left(1, z_{1, i}, \ldots, z_{N_{M}, i}\right)$. Then, since $\mathbf{Z}_{i}$ is a unitary matrix, it follows that

$$
\boldsymbol{\Phi}_{\mathbf{R}_{i}}^{-1}=\mathbf{Z}_{i}\left(E_{s} \boldsymbol{\Phi}_{t}+\left(N_{0}+\sigma_{\mathrm{ICI}}^{2}\right) \mathbf{I}\right)^{-1} \mathbf{Z}_{i}^{\dagger}
$$

Therefore, $\hat{\boldsymbol{\Phi}}_{\mathbf{R}_{i}}^{-1}$ can be obtained by substituting estimated sequence $\hat{\mathbf{Z}}_{i}=\operatorname{diag}\left(1, \hat{z}_{1, i}, \ldots, \hat{z}_{N_{M}, i}\right)$ for $\mathbf{Z}_{i}$ in $(21)$. When the channel is stationary such that all the variables $E_{s}, N_{0}, \Phi_{t}$, and $\sigma_{\text {ICI }}$ remain constant, $\hat{\Phi}_{\mathbf{R}_{i}}^{-1}$ need not be calculated each time.

\subsection{Covariance matrix in frequency-domain MSDD}

Likewise, for FD-MSDD, by noticing that the correlation of interest is irrelevant to the OFDM symbol index $i$, the covariance of $R(l)$ in (7) can be expressed as

$$
\begin{aligned}
& E\left[R(l) R^{*}(l+\alpha)\right] \\
&=E[ a(l) H(l) H^{*}(l+\alpha) a^{*}(l+\alpha) \\
&+a(l) H(l) C^{*}(l+\alpha) \\
&+a^{*}(l+\alpha) H^{*}(l+\alpha) C(l) \\
&\left.+C(l) C^{*}(l+\alpha)+W(l) W^{*}(l+\alpha)\right] .
\end{aligned}
$$

Given the transmitted symbols $a(l),(22)$ can be decomposed as

$$
\begin{aligned}
& E\left[R(l) R^{*}(l+\alpha)\right] \\
&= a(l) E\left[H(l) H^{*}(l+\alpha)\right] a^{*}(l+\alpha) \\
&+E\left[a(l) H(l) C^{*}(l+\alpha)\right]+E\left[a^{*}(l+\alpha) H^{*}(l+\alpha) C(l)\right] \\
&+E\left[C(l) C^{*}(l+\alpha)\right]+E\left[W(l) W^{*}(l+\alpha)\right] .
\end{aligned}
$$

The first term in (23) requires the correlation of the multiplicative distortion, which is given by

$$
\begin{aligned}
\phi_{f}(\alpha) & \equiv E\left[H(l) H^{*}(l+\alpha)\right] \\
& =\frac{1}{N_{s}^{2}}\left(N_{s}+2 \sum_{k=1}^{N_{s}-1}\left(N_{s}-k\right) J_{0}\left(2 \pi f_{D} T_{s} k\right)\right) \sum_{m=0}^{M_{p}-1} \sigma_{m}^{2} e^{j\left(2 \pi \alpha m / N_{s}\right)} \\
& =\left(1-\frac{\sigma_{\mathrm{ICI}}^{2}}{E_{s}}\right) \sum_{m=0}^{M_{p}-1} \sigma_{m}^{2} e^{j\left(2 \pi \alpha m / N_{s}\right)} .
\end{aligned}
$$

Due to the wide-sense stationarity of the fading process, the covariance matrix of $H(l)$ can be given by $\Phi_{f}$ in which the $(\beta, \gamma)$ th element has $\phi_{f}(\gamma-\beta)$ of $(24)$. 
The second term in (23) requires the calculation of the following term:

$$
\begin{aligned}
\kappa_{l}(\beta, \gamma) & =E\left[a(l+\beta) H(l+\beta) C^{*}(l+\gamma)\right] \\
= & \frac{1}{N_{s}^{2}} a(l+\beta) \sum_{n=l, n \neq l+\gamma}^{l+N_{M}} a^{*}(n) \sum_{m=0}^{M_{p}-1} \sigma_{m}^{2} e^{j\left(2 \pi(n-(l+\beta)) m / N_{s}\right)} \\
& \cdot \sum_{k=0}^{N_{s}-1} \sum_{k^{\prime}=0}^{N_{s}-1} J_{0}\left(2 \pi f_{D} T_{s}\left(k-k^{\prime}\right)\right) e^{-j\left(2 \pi k^{\prime}(n-(l+\gamma)) / N_{s}\right)} \\
= & \frac{E_{s}}{N_{s}^{2}} z_{\beta}(l) \sum_{n=l, n \neq l+\gamma}^{l+N_{M}} z_{n-l}^{*}(l) \sum_{m=0}^{M_{p}-1} \sigma_{m}^{2} e^{j\left(2 \pi(n-(l+\beta)) m / N_{s}\right)} \\
& \cdot \sum_{k=0}^{N_{s}-1} \sum_{k^{\prime}=0}^{N_{s}-1} J_{0}\left(2 \pi f_{D} T_{s}\left(k-k^{\prime}\right)\right) e^{-j\left(2 \pi k^{\prime}(n-(l+\gamma)) / N_{s}\right)},
\end{aligned}
$$

where we have applied (8). Using the Taylor series expansion of the Bessel function $J_{0}(2 \pi x) \approx 1-(\pi x)^{2}$, which becomes valid for $|x| \ll 1[15], \kappa_{l}(\beta, \gamma)$ in (25) can be approximated as

$$
\begin{aligned}
& \kappa_{l}(\beta, \gamma) \\
& \approx \\
& \quad \frac{E_{s}\left(\pi f_{D} T_{s}\right)^{2}}{N_{s}} z_{\beta}(l) \\
& \quad \cdot \sum_{n=l, n \neq l+\gamma}^{l+N_{M}} z_{n-l}^{*}(l) \sum_{m=0}^{M_{p}-1} \sigma_{m}^{2} e^{j\left(2 \pi(n-(l+\beta)) m / N_{s}\right)} p(n-(l+\gamma)),
\end{aligned}
$$

where

$$
p(\alpha)=\sum_{k^{\prime}=0}^{N_{s}-1}\left(\left(N_{s}-1\right) k^{\prime}-k^{\prime 2}\right) e^{-j\left(2 \pi k^{\prime} \alpha / N_{s}\right)} .
$$

Likewise, the third term in (23) requires the following:

$$
\begin{aligned}
\xi_{l}(\beta, \gamma) & \\
\equiv & E\left[a^{*}(l+\gamma) H^{*}(l+\gamma) C(l+\beta)\right] \\
= & \frac{E_{s}}{N_{s}^{2}} z_{\gamma}^{*}(l) \sum_{n=l, n \neq l+\beta}^{l+N_{M}} z_{n-l}(l) \sum_{m=0}^{M_{p}-1} \sigma_{m}^{2} e^{-j\left(2 \pi(n-(l+\gamma)) m / N_{s}\right)} \\
& \cdot \sum_{k=0}^{N_{s}-1} \sum_{k^{\prime}=0}^{N_{s}-1} J_{0}\left(2 \pi f_{D} T_{s}\left(k^{\prime}-k\right)\right) e^{-j\left(2 \pi k^{\prime}(-n+l+\beta) / N_{s}\right)} \\
\approx & \frac{E_{s}\left(\pi f_{D} T_{s}\right)^{2}}{N_{s}} z_{\gamma}^{*}(l) \\
& \cdot \sum_{n=l, n \neq l+\beta}^{l+N_{M}} z_{n-l}(l) \sum_{m=0}^{M_{p}-1} \sigma_{m}^{2} e^{-j\left(2 \pi(n-(l+\gamma)) m / N_{s}\right)} p(-n+l+\beta) .
\end{aligned}
$$

The fourth term in (23) corresponds to the ICI, which is given by

$$
\begin{aligned}
& \phi_{C, l}(\beta, \gamma) \\
& \equiv E\left[C(l+\beta) C^{*}(l+\gamma)\right] \\
& =\frac{E_{s}}{N_{s}^{2}} \sum_{n=0, n \neq l, \ldots, l+N_{M}}^{N_{s}-1} \sum_{k=0}^{N_{s}-1} \sum_{k^{\prime}=0}^{N_{s}-1} J_{0}\left(2 \pi f_{D} T_{s}\left(k-k^{\prime}\right)\right) \\
& \cdot e^{j\left(2 \pi k(n-(l+\beta)) / N_{s}\right)} e^{-j\left(2 \pi k^{\prime}(n-(l+\gamma)) / N_{s}\right)} \\
& +\frac{E_{s}}{N_{s}^{2}} \sum_{n=l, n \neq l+\beta}^{l+N_{M}} z_{n-l}(l) \sum_{n^{\prime}=l, n^{\prime} \neq l+\gamma}^{l+N_{M}} z_{n^{\prime}-l}^{*}(l) \\
& \cdot \sum_{m=0}^{M_{p}-1} \sigma_{m}^{2} e^{j\left(2 \pi\left(n^{\prime}-n\right) m / N_{s}\right)} \\
& \cdot \sum_{k=0}^{N_{s}-1} \sum_{k^{\prime}=0}^{N_{s}-1} J_{0}\left(2 \pi f_{D} T_{s}\left(k-k^{\prime}\right)\right) \\
& \cdot e^{j\left(2 \pi k(n-(l+\beta)) / N_{s}\right)} e^{j\left(2 \pi k^{\prime}\left(-n^{\prime}+(l+\gamma)\right) / N_{s}\right)} \\
& \approx \frac{2 E_{s}\left(\pi f_{D} T_{s}\right)^{2}}{N_{s}^{2}}\left\{\sum_{n=0, n \neq l, \ldots, l+N_{M}}^{N_{s}-1} q(n-(l+\beta)) q(-n+(l+\gamma))\right. \\
& +\sum_{n=l, n \neq l+\beta}^{l+N_{M}} z_{n-l}(l) \\
& \sum_{n^{\prime}=l, n^{\prime} \neq l+\gamma}^{l+N_{M}} z_{n^{\prime}-l}^{*}(l) \sum_{m=0}^{M_{p}-1} \sigma_{m}^{2} e^{j\left(2 \pi\left(n^{\prime}-n\right) m / N_{s}\right)} \\
& \left.q(n-(l+\beta)) q\left(-n^{\prime}+(l+\gamma)\right)\right\} \text {, }
\end{aligned}
$$

where

$$
q(\alpha)=\sum_{k=0}^{N_{s}-1} k e^{j\left(2 \pi k \alpha / N_{s}\right)} .
$$

Finally, for the AWGN term, we have

$$
E\left[W(l) W^{*}(l+\alpha)\right]=N_{0} \delta_{0, \alpha} .
$$

In the following, the notations $\mathbf{K}_{l}, \boldsymbol{\Xi}_{l}$, and $\boldsymbol{\Phi}_{C, l}$ represent the matrices with the $(\beta, \gamma)$ th element given by $\kappa_{l}(\beta, \gamma)$, $\xi_{l}(\beta, \gamma)$, and $\phi_{C, l}(\beta, \gamma)$, respectively. Then, using (23), (24), (25), (26), (27), (28), (29), (30), and (31), it can be shown that

$$
\boldsymbol{\Phi}_{\mathbf{R}(l)}=\mathbf{Z}(l)\left(E_{s} \boldsymbol{\Phi}_{f}+N_{0} \mathbf{I}\right) \mathbf{Z}^{\dagger}(l)+\mathbf{K}_{l}+\boldsymbol{\Xi}_{l}+\boldsymbol{\Phi}_{C, l} .
$$

The exact calculation of (32) requires the knowledge of both delay profile and $f_{D}$. Furthermore, it requires higher computational complexity resulting from (25), (26), (27), (28), and (29) and calculations of inverse matrices $\hat{\boldsymbol{\Phi}}_{\mathbf{R}(l)}^{-1}$ over all $\hat{\mathbf{Z}}(l)$. To obviate the computation of these unwieldy terms, we also introduce the following suboptimal alternative:

$$
\hat{\boldsymbol{\Phi}}_{\mathbf{R}(l)}=\hat{\mathbf{Z}}(l)\left(E_{s} \boldsymbol{\Phi}_{f}+\left(N_{0}+\sigma_{\mathrm{ICI}}^{2}\right) \mathbf{I}\right) \hat{\mathbf{Z}}^{\dagger}(l) .
$$


This approximate covariance matrix can be obtained by simply substituting the covariance matrix of the multiplicative distortion $\boldsymbol{\Phi}_{f}$ in FD for $\boldsymbol{\Phi}_{t}$ in (20). Since this approximate covariance matrix has an analogous aspect to the covariance matrix in TD, the required computation can be significantly reduced. The price for this simplification is its performance degradation caused by the time selectiveness of the channel, compared to FD-MSDD with the exact covariance matrix. Note that without ICI, the matrices (32) and (33) become identical. The BER performance of this suboptimal FD-MSDD is examined over both time- and frequencyselective Rayleigh fading channels in Section 5.

\section{BIT ERROR PROBABILITY ANALYSIS}

\subsection{Pairwise error probability}

The PEP of MSDD for OFDM can be derived simply by substituting the covariance matrix derived in Section 3 for that of PEP given in [6]. It can be shown that

$$
\begin{aligned}
P\left(\mathbf{c}_{i}(l) \longrightarrow \hat{\mathbf{c}}_{i}(l)\right) & =\operatorname{Prob}(D \leq 0) \\
& =-\sum \text { Residue }\left[\frac{\Phi_{D}(s)}{s}\right]_{\text {RPpoles }},
\end{aligned}
$$

where

$$
\begin{aligned}
D & =M\left(\hat{\mathbf{c}}_{i}(l)\right)-M\left(\mathbf{c}_{i}(l)\right) \\
& =\mathbf{R}_{i}^{\dagger}(l)\left(\hat{\boldsymbol{\Phi}}_{\mathbf{R}_{i}(l)}^{-1}-\boldsymbol{\Phi}_{\mathbf{R}_{i}(l)}^{-1}\right) \mathbf{R}_{i}(l),
\end{aligned}
$$

$\Phi_{D}(s)$ is the characteristic function of $D$, and the summation is taken over all the residues calculated at the poles of $\Phi_{D}(s) / s$ located on the right-hand plane. Following [6], one may have

$$
\Phi_{D}(s)=\prod_{k=1}^{N_{M}+1} \frac{1}{2 \lambda_{k} s+1},
$$

where $\lambda_{k}$ is the $k$ th eigenvalue of the matrix

$$
\mathbf{G}=\boldsymbol{\Phi}_{\mathbf{R}_{i}(l)}\left(\hat{\boldsymbol{\Phi}}_{\mathbf{R}_{i}(l)}^{-1}-\boldsymbol{\Phi}_{\mathbf{R}_{i}(l)}^{-1}\right) .
$$

This expression is the exact PEP of TD-MSDD and FDMSDD. The PEP of the suboptimal FD-MSDD can be obtained simply by replacing the covariance matrix $\hat{\boldsymbol{\Phi}}_{\mathbf{R}_{i}(l)}$ in (37) with the corresponding covariance matrix in (33). The covariance matrix $\Phi_{\mathbf{R}_{i}(l)}$ in (37) remains unchanged and it corresponds to the exact covariance matrix associated with the actual received symbols.

\subsection{Approximate BER}

The information symbol sequence $c_{i}(l)$ has $N_{M} \log _{2} M$ information bits denoted by $\mathbf{u}_{i}(l)$. Let $\hat{\mathbf{u}}_{i}(l)$ also denote estimated information bits associated with $\hat{\mathbf{c}}_{i}(l)$. The pairwise BER associated with transmitting a sequence $\mathbf{c}_{i}(l)$ and detecting an erroneous sequence $\hat{\mathbf{c}}_{i}(l)$ is given by

$$
\begin{aligned}
P_{b} & \left(\mathbf{c}_{i}(l) \longrightarrow \hat{\mathbf{c}}_{i}(l)\right) \\
\quad & =\frac{1}{N_{M} \log _{2} M} h\left(\mathbf{u}_{i}(l), \hat{\mathbf{u}}_{i}(l)\right) P\left(\mathbf{c}_{i}(l) \longrightarrow \hat{\mathbf{c}}_{i}(l)\right),
\end{aligned}
$$

where $h\left(\mathbf{u}_{i}(l), \hat{\mathbf{u}}_{i}(l)\right)$ denotes the Hamming distance between $\mathbf{u}_{i}(l)$ and $\hat{\mathbf{u}}_{i}(l)$.

An upper bound on the BER can be obtained by the union of all pairwise error events. The BER of TD-MSDD is independent of the OFDM symbol index $i$, the subcarrier index $l$, and information symbol sequence $\mathrm{c}$ in terms of theoretical BER associated with the corresponding covariance matrix (20). As a result, c can be assumed as the all-zerophase sequence, that is, $\mathbf{c}=(1, \ldots, 1)$. The union bound on the BER of TD-MSDD can then be written as

$$
\begin{aligned}
P_{b} & \leq \sum_{\hat{\mathbf{c}} \neq \mathbf{c}} P_{b}(\mathbf{c} \longrightarrow \hat{\mathbf{c}}) \\
& =\frac{1}{N_{M} \log _{2} M} \sum_{\hat{\mathbf{c}} \neq \mathbf{c}} h(\mathbf{u}, \hat{\mathbf{u}}) P(\mathbf{c} \longrightarrow \hat{\mathbf{c}}),
\end{aligned}
$$

where the summation is taken over all the distinct sequences $\hat{c}$ which differ from the transmitted information symbol sequence $\mathbf{c}$. On the other hand, the BER of both the optimal and suboptimal FD-MSDD is dependent on the transmitted sequence c. Since it is independent of the subcarrier index $l$, $l$ can be assumed to be 0 . It must be averaged over all the sequences $\mathbf{c}$. The union bound on the BER of FD-MSDD can then be obtained as

$$
\begin{aligned}
P_{b} & \leq \frac{1}{M^{N_{M}}} \sum_{\mathbf{c}} \sum_{\hat{\mathbf{c}} \neq \mathbf{c}} P_{b}(\mathbf{c} \longrightarrow \hat{\mathbf{c}}) \\
& =\frac{1}{M^{N_{M}} N_{M} \log _{2} M} \sum_{\mathbf{c}} \sum_{\hat{\mathbf{c}} \neq \mathbf{c}} h(\mathbf{u}, \hat{\mathbf{u}}) P(\mathbf{c} \longrightarrow \hat{\mathbf{c}}) .
\end{aligned}
$$

Direct application of (39) and (40), however, does not yield a tight bound of the bit error performance for TDMSDD and FD-MSDD over time- and frequency-selective Rayleigh fading channels. As shown in [6] for single-carrier transmission over the time-selective channel, the BER can be approximated by the summation of the PEP over the set of most likely error events. These most likely error events are determined by the set $\left\{\hat{z}_{1}, \ldots, \hat{z}_{N_{M}}\right\}$ which has the highest correlation with the set $\left\{z_{1}, \ldots, z_{N_{M}}\right\}$, where the correlation is defined as $\mu=\left|1+\sum_{k=1}^{N_{M}} z_{k} \hat{z}_{k}\right|^{2}$. There are only a total of 2 for $N_{M}=1$ and $2 N_{M}+2$ for $N_{M} \geq 2$ such events over each set $\left\{z_{1}, \ldots, z_{N_{M}}\right\}$. Since the difference of PEP between TDMSDD and MSDD for single-carrier transmission is only an additive ICI, the BER of TD-MSDD can be approximated by the same method. In the case of FD-MSDD, when the effects of the ICI are relatively small, the covariance matrix of FDMSDD is similar to that of TD-MSDD. Hence, we conjecture that the BER of FD-MSDD can be also approximated by the same method. Consequently, by defining the set of these most likely error events as $\chi$, the approximate BER can be expressed as

$$
\begin{aligned}
& P_{b} \approx \frac{1}{N_{M} \log _{2} M} \sum_{\hat{\mathbf{c}} \neq \mathbf{c}, \hat{\mathbf{c}} \in \chi} h(\mathbf{u}, \hat{\mathbf{u}}) P(\mathbf{c} \longrightarrow \hat{\mathbf{c}}), \\
& \quad \text { for TD-MSDD, } \\
& P_{b} \approx \frac{1}{M^{N_{M} N_{M} \log _{2} M} \sum_{\mathbf{c}} \sum_{\hat{\mathbf{c}} \neq \mathbf{c}, \hat{\mathbf{c}} \in \chi} h(\mathbf{u}, \hat{\mathbf{u}}) P(\mathbf{c} \longrightarrow \hat{\mathbf{c}}),}
\end{aligned}
$$




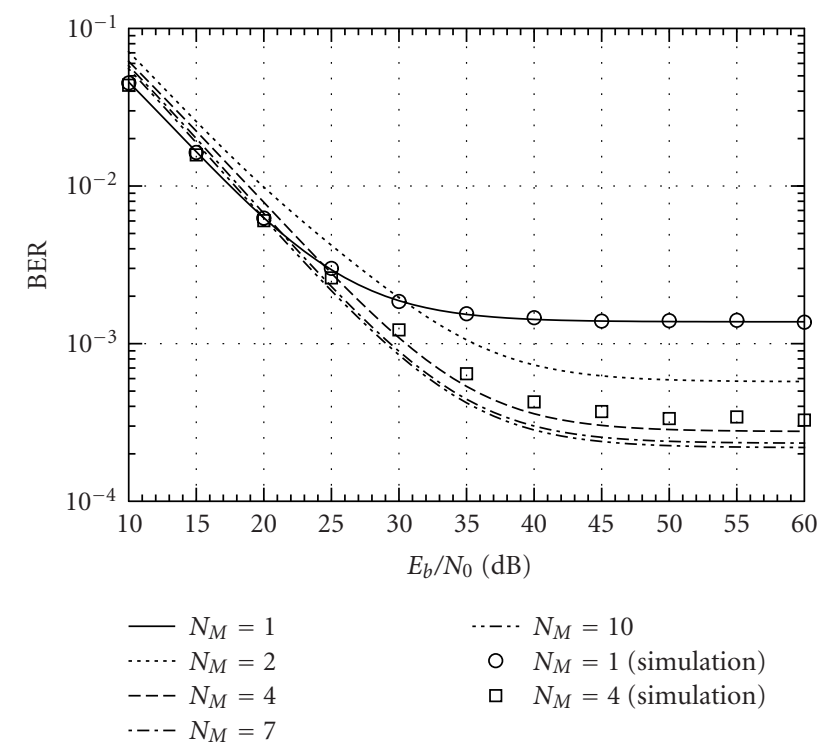

FIGURE 2: BER performance of TD-MSDD with QDPSK over the time- and frequency-selective Rayleigh fading channel with $\overline{f_{D}}=$ $0.01, \overline{T_{m}} \leq 7 / 64, R_{G}=7 / 64 . N_{M}=1$ corresponds to conventional DD.

It is shown in [8] that for TD-DD and FD-DD (i.e., $N_{M}=1$ ) with QDPSK, inphase and quadrature components of the received sequence are statistically independent. Thus, in the case of TD-DD and FD-DD with QDPSK, most likely error events are statistically independent, and thus the BER obtained by the above method results in a closed-form expression.

\section{NUMERICAL RESULTS}

Numerical results presented in this section include Monte Carlo simulation results and theoretical results based on the approximate BER in (41). These results are investigated over a two-ray equal-power profile. As a generalization of MSDD to OFDM, we normalize the Doppler frequency and delay spread by the OFDM symbol period, defined as $\overline{f_{D}}=f_{D} N_{s} T_{s}$ and $\overline{T_{m}}=M_{p} T_{s} /\left(N_{s} T_{s}\right)=M_{p} / N_{s}$, respectively. For this channel, the average power of the $m$ th channel tap can be expressed as

$$
\sigma_{m}^{2}= \begin{cases}\frac{1}{2}, & \text { for } m=0, M_{p} \\ 0, & \text { for } m \neq 0, M_{p}\end{cases}
$$

\subsection{Verification of analysis}

Theoretical and simulation results for the BER performance of TD-MSDD with QDPSK over the time- and frequencyselective channel with $\overline{f_{D}}=0.01, \overline{T_{m}} \leq 7 / 64$, guard interval ratio $R_{G}=7 / 64$ (defined as $R_{G}=G / N_{s}$ ), are shown in

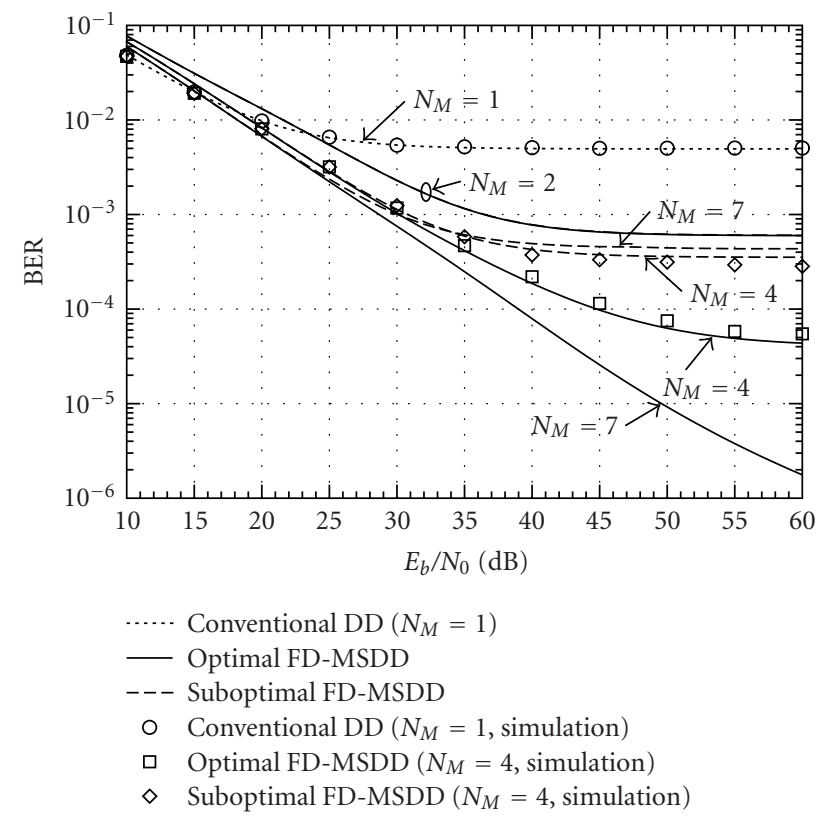

FIGURE 3: BER performance of FD-MSDD with QDPSK over the time- and frequency-selective Rayleigh fading channel with $\overline{f_{D}}=$ $0.01, \overline{T_{m}}=2 / 64, N_{s}=64, G \geq 2$.

Figure 2. Note that the OFDM system with $N_{s}=64$, a carrier frequency of $5 \mathrm{GHz}$, a bandwidth of $1 \mathrm{MHz}$, and a mobile station velocity of $34 \mathrm{~km} / \mathrm{h}$ may result in $\overline{f_{D}} \approx 0.01$. In this case, since the ISI does not occur, these results are independent of the specific value of $\overline{T_{m}}(\leq 7 / 64)$. Although $R_{G}$ is relevant to the correlation of the multiplicative distortion, its effect is relatively small without ISI. It is observed from Figure 2 that for $N_{M}=4$, the simulation results show close agreement with the theoretical results at high SNR (above $25 \mathrm{~dB}$ ). At lower SNR, however, the approximation appears to be slightly pessimistic, due to the asymptotic tightness nature of the union bound. The performance degradation of TD-DD is noticeable over the time-selective channel. This is caused by both decrease in the intersymbol correlation of the multiplicative distortion and the irreducible ICI associated with the OFDM transmission. Even though increasing $N_{M}$ in TD-MSDD may alleviate performance degradation due to decrease in the intersymbol correlation, it is not capable of reducing the effect of the ICI. Thus, the error floor appears for TD-MSDD even with large $N_{M}$.

Figure 3 compares theoretical and simulation results for the BER performance of FD-MSDD with QDPSK over the time- and frequency-selective channel with $\overline{f_{D}}=0.01, \overline{T_{m}}=$ $2 / 64, N_{s}=64, G \geq 2$. Note that the result is irrelevant to the value of $N_{s}$. Similar to the case of TD-MSDD, good agreement between the simulation and theoretical results is observed at high SNR (above $20 \mathrm{~dB}$ ). Even though the performance degradation is noticeable for FD-DD, increasing $N_{M}$ may improve the bit error performance of both the optimal and suboptimal FD-MSDD. Furthermore, the significant benefit of the optimal FD-MSDD over the suboptimal FDMSDD is apparent. This stems from the fact that the optimal 


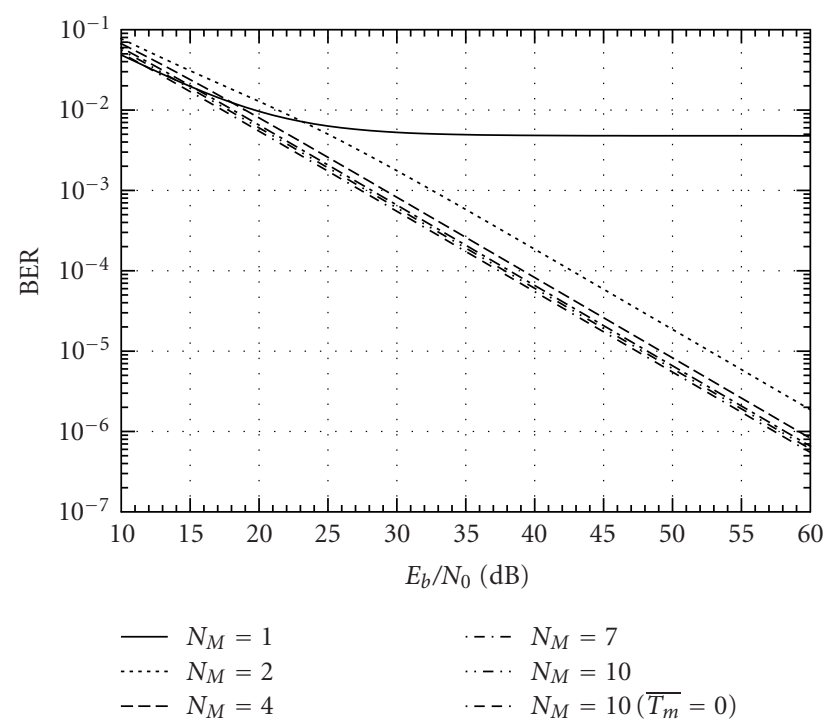

FIGURE 4: BER performance of FD-MSDD with QDPSK over the time-nonselective (i.e., $\overline{f_{D}}=0.0$ ) frequency-selective Rayleigh fading channel with $\overline{T_{m}}=2 / 64, G \geq M_{p}$.

metric calculates the exact impact of ICI whereas the suboptimal metric only utilizes the approximation.

\subsection{Asymptotic performance of FD-MSDD}

Figure 4 shows theoretical results for the BER performance of FD-MSDD with QDPSK over the time-nonselective (i.e., $\left.\overline{f_{D}}=0.0\right)$ frequency-selective channel with $\overline{T_{m}}=2 / 64$ and $G \geq M_{p}$. In this case, the behavior of optimal FD-MSDD is equivalent to that of suboptimal FD-MSDD, since $\mathbf{K}_{l}, \boldsymbol{\Xi}_{l}$, $\boldsymbol{\Phi}_{C, l}$ in (32) are all equal to zero matrices. It is observed from Figure 4 that without ICI, the irreducible error floor associated with a decrease in the inter-subcarrier correlation of the multiplicative distortion for FD-DD can be efficiently eliminated for FD-MSDD even with $N_{M}$ as small as 2. When $N_{M}=10$, the performance degradation from that with frequency-nonselective channel is approximately $0.4 \mathrm{~dB}$ at a BER of $10^{-6}$. Thus, in the limit as the observation interval approaches infinity, the BER behavior of FD-MSDD over frequency-selective channels without ICI approaches that with the same observation interval over a static channel.

\subsection{Comparison between TD-MSDD and FD-MSDD}

Figure 5 shows theoretical results for the BER performance of TD-DD and FD-DD with QDPSK employed in each dimension and $R_{G}=7 / 64$. For the sake of comparison of the asymptotic bit error performance at error floor region, $E_{b} / N_{0}$ is fixed at $60[\mathrm{~dB}]$. Note that, given the system parameters by $N_{s}=64,5 \mathrm{GHz}$ carrier frequency, and $1 \mathrm{MHz}$ bandwidth, the range $\overline{f_{D}}$ up to 0.05 corresponds to the mobile station velocity up to approximately $170 \mathrm{~km} / \mathrm{h}$. It is observed from Figure 5 that the performance degradation of TD-DD is caused only by the time selectiveness and is irrelevant to the frequency selectiveness, as long as the ISI is

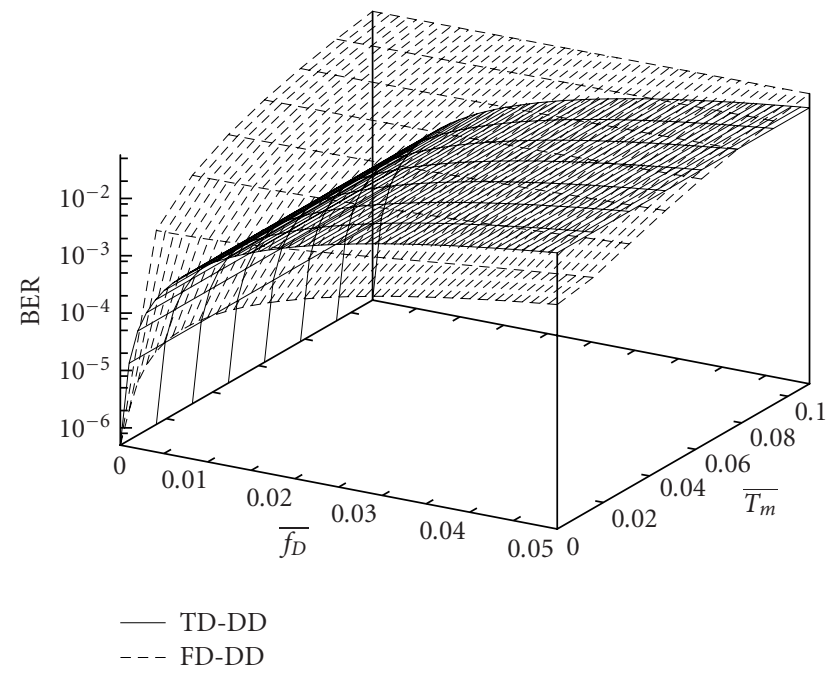

FIGURE 5: BER performance of TD-DD and FD-DD with QDPSK in each dimension, $E_{b} / N_{0}=60(\mathrm{~dB}), R_{G}=7 / 64$.

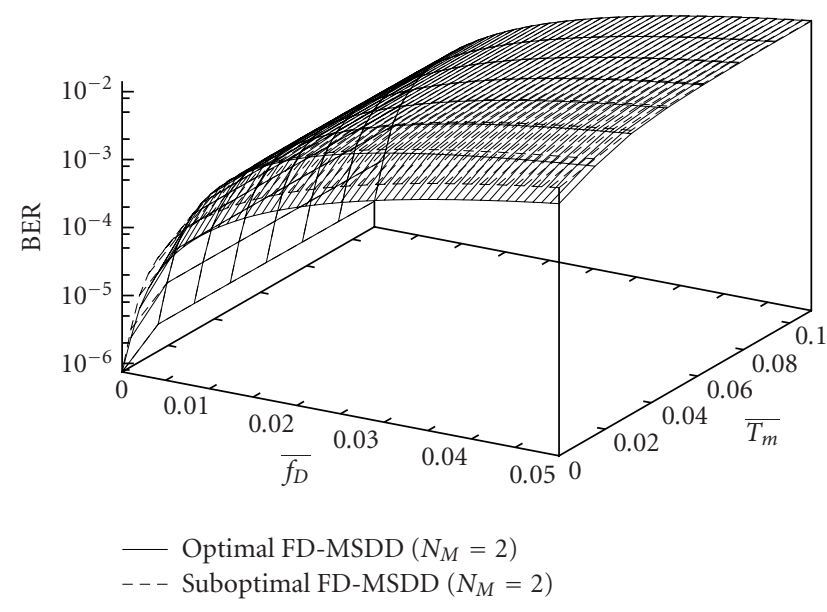

FIGURE 6: BER performance of optimal FD-MSDD and suboptimal FD-MSDD with QDPSK in each dimension, $E_{b} / N_{0}=60(\mathrm{~dB})$, $N_{M}=2, N_{s}=64, G=7$.

negligible. For FD-DD, the frequency selectiveness is the limiting factor for the BER. These results suggest the importance of appropriate selection of the DD technique matched to the channel statistics.

Theoretical results for the BER performance of the optimal and suboptimal FD-MSDD with QDPSK in each dimension with $E_{b} / N_{0}=60[\mathrm{~dB}], N_{M}=2, N_{s}=64, G=7$ are shown in Figure 6, where it is observed that for $N_{M}=2$, the difference between the optimal and suboptimal FD-MSDD is negligible. Thus, the optimal FD-MSDD with complicated decision metric may not be necessarily rewarding in practice. Unlike FD-DD, both the FD-MSDD approaches are robust against the frequency selectiveness, and the ICI due to the time selectiveness is the limiting factor. 


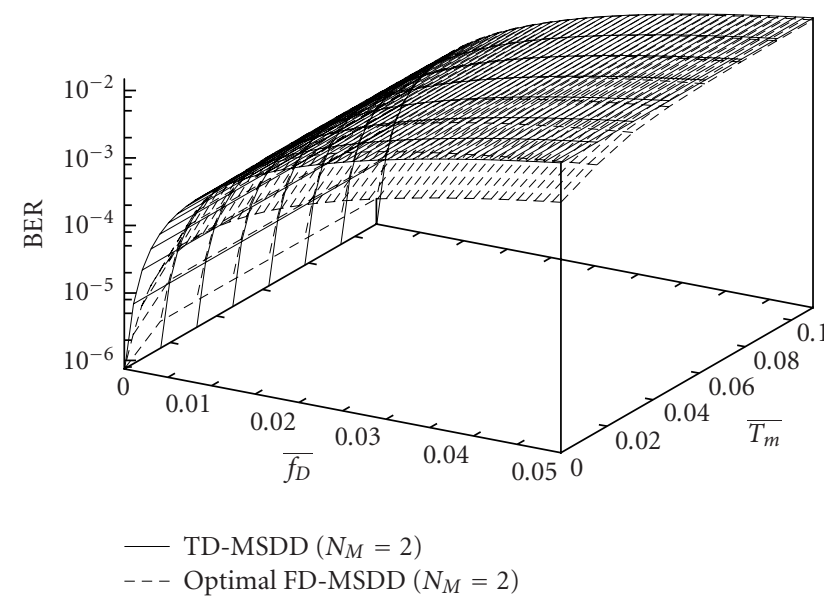

FIGURE 7: BER performance of TD-MSDD and optimal FD-MSDD with QDPSK in each dimension, $E_{b} / N_{0}=60(\mathrm{~dB}), N_{M}=2, N_{s}=$ $64, G=7$.

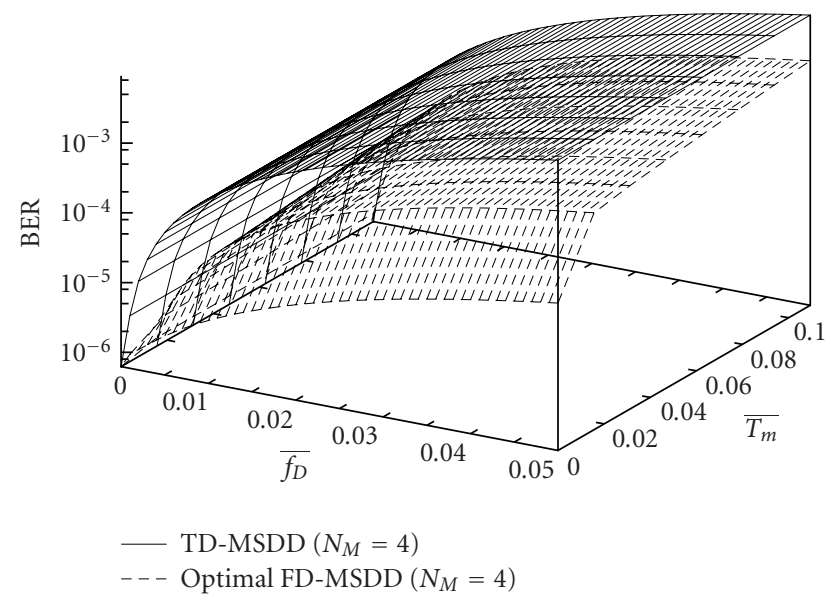

FIGURE 8: BER performance of TD-MSDD and optimal FD-MSDD with QDPSK in each dimension, $E_{b} / N_{0}=60(\mathrm{~dB}), N_{M}=4, N_{s}=$ $64, G=7$.

Theoretical results for the BER performance of TDMSDD and the optimal FD-MSDD with the same channel and system parameters above are shown in Figure 7. It is observed that for $N_{M}=2$, the behavior of FD-MSDD is analogous to that of TD-MSDD, since both are able to mitigate the performance degradation associated with the decrease in the correlation of the multiplicative distortion. With $N_{M}=2$, however, they do not alleviate the effect of ICI.

Figure 8 shows the performance of the system with the same parameters as Figure 7 except now we set $N_{M}=4$. It is observed that even though the optimal FD-MSDD requires higher complexity, it outperforms TD-MSDD on almost all channel statistics compared. This difference comes from the fact that the optimal FD-MSDD can also mitigate the ICI.

Finally, theoretical results for the BER performance of TD-MSDD and the suboptimal FD-MSDD with the same

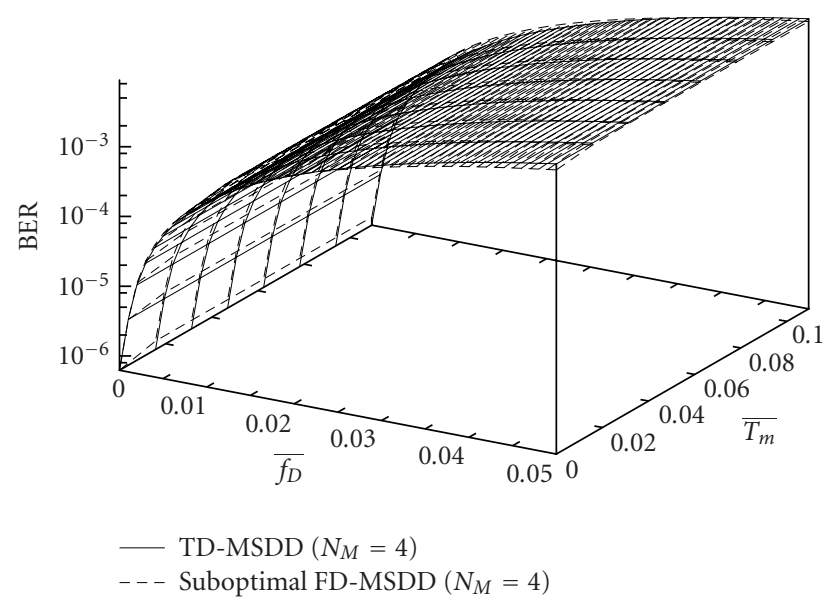

FIGURE 9: BER performance of TD-MSDD and suboptimal FDMSDD with QDPSK in each dimension, $E_{b} / N_{0}=60(\mathrm{~dB}), N_{M}=4$, $N_{s}=64, G=7$.

conditions as Figure 8 are shown in Figure 9, where it is observed that the behavior of the suboptimal FD-MSDD is analogous to that of TD-MSDD. Thus, for $N_{M} \geq 2$, the difference between the BER performance of TD-MSDD and that of the suboptimal FD-MSDD may be negligible.

\section{CONCLUSION}

In this paper, we applied MSDD to OFDM over time- and frequency-selective Rayleigh fading channels under the assumption that the guard time is longer than the delay spread, thus causing no effective ISI. Optimal decision metrics of TD-MSDD and FD-MSDD have been derived based on the exact covariance matrix conditioned on transmitted information symbol sequence. The theoretical BER performance of MSDD for OFDM has been analyzed, and based on these analytical results, we have shown that when simple receiver structure is preferable, both TD-MSDD and the suboptimal FD-MSDD provide a good performance because of their robustness against the time- and frequency-selective nature of the channel. Thus, as opposed to need of careful selection between TD-DD and FD-DD according to the channel statistics, the difference in BER performance between TDMSDD and the suboptimal FD-MSDD is negligible. Furthermore, it has been shown that if the enhancement of computational complexity at the receiver is acceptable, the optimal FD-MSDD may be a very effective strategy due to its robustness against the ICI over such channels.

In the limit as the observation interval approaches infinity, the BER performance of FD-MSDD over frequencyvarying channels without ICI may approach that with the same observation interval over a static channel. However, the high computational complexity is the main disadvantage of MSDD, and it has been shown in $[16,17]$ that decision-feedback differential detection (DF-DD) techniques provide a good performance at a low computational complexity. Since it has been shown that MSDD and DF-DD are 
equivalent and DF-DD can be derived from MSDD by introducing decision-feedback symbols into the MSDD metrics, the metrics proposed in this paper can be also applied to DFDD for OFDM for reduction of computational complexity. Therefore, extension of the proposed metric to DF-DD with OFDM may be a topic for future study.

\section{REFERENCES}

[1] Y. Wu and B. Caron, "Digital television terrestrial broadcasting," IEEE Communications Magazine, vol. 32, no. 5, pp. 4652, 1994.

[2] R. van Nee and R. Prasad, OFDM for Wireless Multimedia Communications, Artech House Publishers, Boston, Mass, USA, 2000.

[3] M. Russell and G. L. Stuber, "Interchannel interference analysis of OFDM in a mobile environment," in IEEE 45th Vehicular Technology Conference (VTC '95), vol. 2, pp. 820-824, Chicago, Ill, USA, July 1995.

[4] D. Divsalar and M. K. Simon, "Multiple-symbol differential detection of MPSK," IEEE Trans. Communications, vol. 38, no. 3, pp. 300-308, 1990.

[5] P. Ho and D. Fung, "Error performance of multiple symbol differential detection of PSK signals transmitted over correlated Rayleigh fading channels," in Proc. IEEE International Conference on Communications (ICC'91), vol. 2, pp. 568-574, Denver, Colo, USA, June 1991.

[6] P. Ho and D. Fung, "Error performance of multiple-symbol differential detection of PSK signals transmitted over correlated Rayleigh fading channels," IEEE Trans. Communications, vol. 40, no. 10, pp. 1566-1569, 1992.

[7] H. Schubert, A. Richter, and K. Iversen, "Differential modulation for OFDM in frequency vs. time domain," in ACTS Mobile Communication Summit, pp. 763-768, Aalborg, Denmark, October 1997.

[8] M. Lott, "Comparision of frequency and time domain differential modulation in an OFDM system for wireless ATM," in IEEE 49th Vehicular Technology Conference (VTC '99), vol. 2, pp. 877-883, Houston, Tex, USA, May 1999.

[9] M. Lott, P. Seidenberg, and S. Mangold, "BER characteristic for the broadband radio channel at $5.2 \mathrm{GHz}$," in Proc. ITG-Workshop Wellenausbreitung bei Funksystemen und Mikrowellensystemen, pp. 53-60, Oberpfaffenhofen, Germany, May 1998.

[10] M. Okada, S. Hara, and N. Morinaga, "Bit error rate performances of orthogonal multicarrier modulation radio transmission systems," IEICE Transaction on Communications, vol. E76-B, pp. 113-119, 1993.

[11] R. F. H. Fischer, L. H.-J. Lampe, and S. H. Muller-Weinfurtner, "Coded modulation for noncoherent reception with application to OFDM," IEEE Trans. Vehicular Technology, vol. 50, no. 4, pp. 910-919, 2001.

[12] J. G. Proakis, Digital Communications, McGraw-Hill, New York, NY, USA, 4th edition, 2000.

[13] W. C. Jakes Jr., Ed., Microwave Mobile Communications, John Wiley \& Sons, New York, NY, USA, 1974.

[14] Y. H. Kim, I. Song, H. G. Kim, T. Chang, and H. M. Kim, "Performance analysis of a coded OFDM system in time-varying multipath Rayleigh fading channels," IEEE Trans. Vehicular Technology, vol. 48, no. 5, pp. 1610-1615, 1999.

[15] W. C. Lee, Mobile Communications Engineering, McGrawHill, New York, NY, USA, 1982.

[16] F. Adachi and M. Sawahashi, "Decision feedback multiplesymbol differential detection for $M$-ary DPSK," Electronics Letters, vol. 29, no. 15, pp. 1385-1387, 1993.
[17] R. Schober, W. H. Gerstacker, and J. B. Huber, "Decisionfeedback differential detection of MDPSK for flat Rayleigh fading channels," IEEE Trans. Communications, vol. 47, no. 7, pp. 1025-1035, 1999.

Akira Ishii received the B.E. and M.E. degrees in communication engineering from the University of Electro-Communications, Tokyo, Japan, in 2002 and 2004, respectively. He has joined NTT DoCoMo, Tokyo, Japan, in 2004. His current research interests include modulation and coding techniques in mobile communications.

Hideki Ochiai received the B.E. degree in communication engineering from Osaka University, Osaka, Japan, in 1996, and the M.E. and Ph.D. degrees in information and communication engineering from The University of Tokyo, Tokyo, Japan, in 1998 and 2001, respectively. From 1994 to 1995 , he was with the Department of Electrical Engineering, University of California (UCLA), Los Angeles, under the scholarship of the

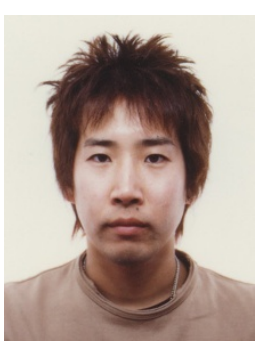
Ministry of Education, Science, and Culture. From 2001 to 2003, he was with the Department of Information and Communication Engineering, The University of Electro-Communications, Tokyo, Japan. Since April 2003, he has been with the Division of Physics, Electrical and Computer Engineering, Yokohama National University, Yokohama, Japan, where he is an Assistant Professor. His current research interests include modulation and coding techniques in mobile communications. Dr. Ochiai was a recipient of a Student Paper Award from the Telecommunications Advancement Foundation in 1999 and the Ericsson Young Scientist Award in 2000.

Tadashi Fujino was born in Osaka, Japan, on 15 July, 1945. He received his B.E. and M.E. degrees in electrical engineering and his Ph.D. degree in communication engineering from Osaka University in 1968, 1970, and 1985, respectively. Since April 2000, he has been Professor in wireless communication at the Department of Information and Communication Engineering, the University of Electro-Communications,

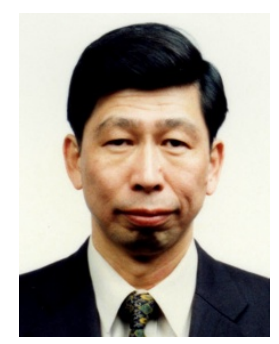
Tokyo, Japan. Before he engaged with the University, he had been engaged with Mitsubishi Electric Corporation, Tokyo, Japan, since 1970, where he devoted his efforts to R\&D in the area of wireless communications such as digital satellite communications and land mobile communications. His major works are the development of $120 \mathrm{Mbit} / \mathrm{s}$ QPSK modem, the Trellis Coded 8-PSK Modem to operate at $120 \mathrm{Mbit} / \mathrm{s}$, and portable phones used in Japan and Europe. He received the Meritorious Award from the ARIB (the Associate of Radio Industries and Businesses of Japan) of MPT of Japan, in 1997. He is an IEEE Fellow. He is also a Member of IEICE and a Member of Society of Information Theory and Its Applications. 